\title{
Offset Cross-Slot-Coupled Dielectric Resonator Antenna for Circular Polarization
}

\author{
Georgios Almpanis, Cristophe Fumeaux, Member, IEEE, and Rüdiger Vahldieck, Fellow, IEEE
}

\begin{abstract}
A cross-slot-coupled cylindrical dielectric resonator antenna (DRA) is studied theoretically and experimentally. In previous papers, a cross-slot of unequal slot lengths was centered under the dielectric resonator (DR), resulting in circular polarized operation of the antenna. In the present study, the design is enhanced by setting the centers of the two slots at different positions and taking into consideration the partial independence of the slot modes from the DRA mode. Thus, circular polarization (CP) bandwidth of up to $4.7 \%$ is attained experimentally in the broadside direction. It is also shown that a largely asymmetrical structure results in a very high bandwidth, but with the tradeoff of distorted CP operation off-broadside.
\end{abstract}

Index Terms-Circular polarization (CP), cross-slot, dielectric resonator antenna (DRA), slot coupling.

\section{INTRODUCTION}

$\mathbf{T}$ HE dielectric resonator antenna (DRA) was first introduced by Long et al. [1] in 1983 and since then it has been extensively studied. The features that make DRAs such an attractive candidate for a number of applications include their high radiation efficiency, compact size, low cost, light weight, and versatility in their shape and feeding mechanism.

The operation of circular polarized DRAs has been the focus of a number of papers in the recent past [2]-[7]. Circular polarization (CP) operation was achieved through complex geometries of the DRA [2], [3] or through the attachment of parasitic patches [4], [5]. In these cases, the complicated shapes of the DRAs, the metallic strips or even the arrays of simple-shaped DRAs increased their manufacturing difficulty and thus their cost and in some cases also their size and weight. In [6], another design was proposed, in which $\mathrm{CP}$ operation was accomplished by using two crossed slots of unequal lengths to couple energy from a microstrip line to a simple cylindrical DRA. Both slots were angled at $45^{\circ}$ with respect to the feeding microstrip line and their centers were at the same position, centered underneath the dielectric resonator (DR), as depicted in the inset of Fig. 1. Their lengths were unequal, so that two near-degenerate orthogonal modes of equal amplitude and $90^{\circ}$ phase difference were excited at frequencies close to that of the fundamental $\mathrm{HEM}_{11 \delta}$ [8] mode of the DRA. The resulting CP bandwidth was 3.91\%.

This letter proposes a new design of a cross-slot coupled DRA, taking into consideration that the slot and the DRA resonances are two partially independent mechanisms [9]. The new design is illustrated in Fig. 1. Each slot is resonant at a particular

Manuscript received February 6, 2006; revised April 26, 2006. This work was supported by ETH Research Grant TH-38/04-1.

The authors are with the Laboratory for Electromagnetic Fields and Microwave Electronics, ETH Zurich, Switzerland (e-mail: almpanis@ ifh.ee.ethz.ch).

Digital Object Identifier 10.1109/LMWC.2006.879484

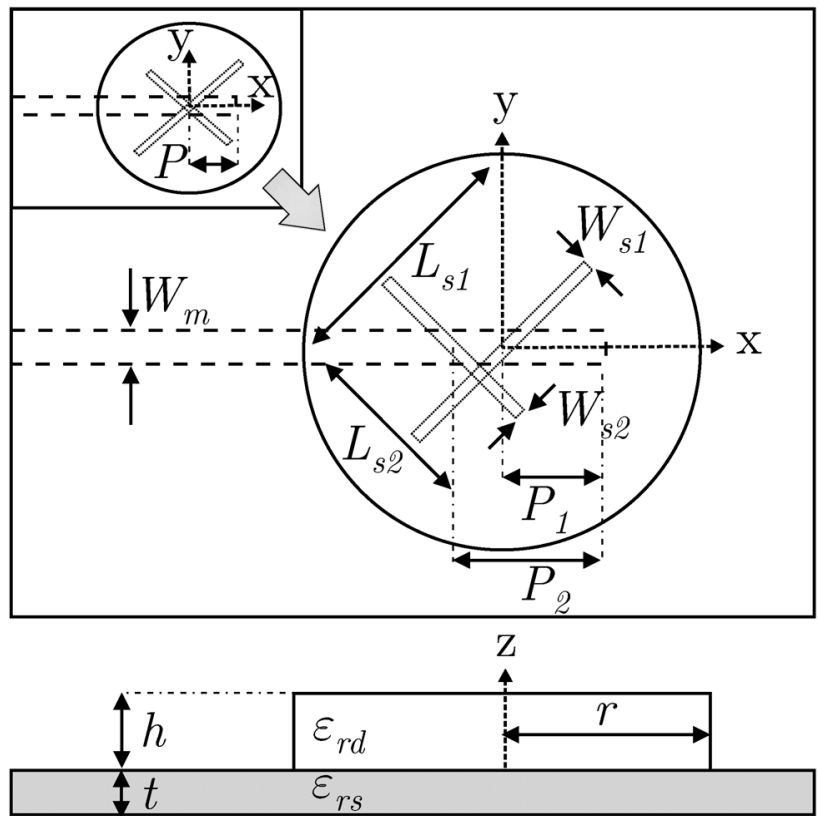

Fig. 1. Schematic of the new cross-slot-coupled DRA. Inset: centered design in [6].

frequency, which depends on its length as well as the permittivity of sub- and superstrate (DRA). The $90^{\circ}$ angle crossing of the slots prevents the coupling between the two excited orthogonal modes and therefore, the investigation for each slot resonance may even be conducted separately. $\mathrm{CP}$ operation for the DRA is obtained when the two modes have equal amplitudes and $\pm 90^{\circ}$ phase difference (for right-handed or left-handed circular polarization). The amplitude of the orthogonal modes is affected by the matching of each slot and consequently also by the stub length (distance between the center of the slot and the open-end of the microstrip line as depicted by $P_{1}, P_{2}$ in Fig. 1). Therefore, excitation by two crossed slots may provide CP operation over a significantly expanded bandwidth, provided the following requirements are satisfied. First, the length of one slot must be adequately larger than that of the other in order to excite modes at different frequencies. Second, a different stub length of the feeding microstrip line must be chosen for every slot so that better matching can be ensured and thus near-degenerate modes will be excited.

\section{DESIGN CONCEPT AND ANTENNA CONFIGURATION}

As discussed before, the slot lengths and the permittivity of the sub- and superstrate (DRA) determine the frequencies of the slot resonances, while the DRA modes depend on the DR dimensions, permittivity, as well as the feeding mechanism. For 
instance, the fundamental DRA mode $\mathrm{HEM}_{11 \delta}$ can be excited by means of a slot at or near the center of the disk or with a probe close to its periphery.

The procedure used to design the circularly polarized cylindrical DRA is based on the concept of partial independence of the resonances. The design for a specified center frequency $f_{\mathrm{cp}}$ of CP operation is made in two main steps.

First, the longer of the two slots is assigned to be resonant at a frequency $f_{1}=f_{\mathrm{cp}}-f^{\prime}$, while the shorter one is at a frequency $f_{2}=f_{\mathrm{cp}}+f^{\prime \prime}$, where $f^{\prime} \approx f^{\prime \prime} \ll f_{\mathrm{cp}}$. To that end, the two slots are designed and optimized separately from one another. Each slot forms an angle of $45^{\circ}$ with respect to the microstrip line and is sandwiched between the substrate and a superstrate of the same height $h$ and permittivity $\varepsilon_{\mathrm{rd}}$ as the DRA. Infinite lateral dimensions are assumed, in order to avoid resonances resulting from the finite dimensions of the structure. The structure can then be analyzed efficiently by means of Ansoft Designer to obtain optimum slot and stub lengths for the desired resonant frequencies for each slot separately. The first slot, with optimum length $L_{s 1}$ and stub length $P_{1}$, is resonant at frequency $f_{1}$ and is forming an angle of $45^{\circ}$ with respect to the microstrip line. Similarly, the second slot of length $L_{s 2}$ and stub length $P_{2}$ is at an angle of $-45^{\circ}$ with respect to the microstrip line and is resonant at frequency $f_{2}$. The two slots are now put together over the microstrip, while retaining the same angle and stub lengths as before. It turns outs that the overall performance of the structure is almost identical to the superposition of the performance of the two slots, since they are crossed at a $90^{\circ}$ angle and therefore no coupling of energy occurs between them.

In the second step, the design of the two crossed slots is kept unchanged but the superstrate is replaced by a cylinder of the same height and permittivity as before. Additionally, the lateral substrate dimensions are made finite. The following simulations are now performed with Ansoft HFSS to accommodate the finite lateral dimensions. The radius of the cylinder is initially chosen such that the frequency of the fundamental DRA $\mathrm{HEM}_{11 \delta}$ mode is $f_{\mathrm{cp}}$. However, in order to obtain optimum CP operation for the DRA, its radius might need to be altered slightly. Further fine-tuning might still be necessary, in order to enhance the CP bandwidth without deteriorating the return loss $(S 11)$ or the radiation patterns. This optimization involves mainly the stub lengths and the position of the disc center relative to the center line of the feeding microstrip.

For a CP operation of the DRA at $5.7 \mathrm{GHz}$, the aforementioned procedure results in an antenna geometry, as illustrated in Fig. 1. The dielectric disc is made from Rogers TMM 10i laminate, with dielectric permittivity $\varepsilon_{\mathrm{rd}}=9.8$, height $h=$ $5.1 \mathrm{~mm}$ and radius $r=15.2 \mathrm{~mm}$. The DR lies on top of the crossed slots which are etched in the finite ground plane of a carrier substrate $\left(\varepsilon_{\mathrm{rs}}=2.2\right.$ and thickness $\left.t=0.7874 \mathrm{~mm}\right)$ with dimensions $120 \mathrm{~mm} \times 120 \mathrm{~mm}$. The center of the DR is exactly on top of the center of the slot with length $L_{s 1}$. This substrate carries on the opposite side the $50 \Omega$ microstrip line with $W_{m}=2.4 \mathrm{~mm}$. The dimensions of the crossed slots are $L_{s 1}=11.4 \mathrm{~mm}, L_{s 2}=8.9 \mathrm{~mm}$ and $W_{s 1}=W_{s 2}=0.3 \mathrm{~mm}$. Slotlines and microstrip line are angled at $45^{\circ}$ and the stub lengths are $P_{1}=4.2 \mathrm{~mm}$ and $P_{2}=4.7 \mathrm{~mm}$ to ensure good impedance matching of the slots.

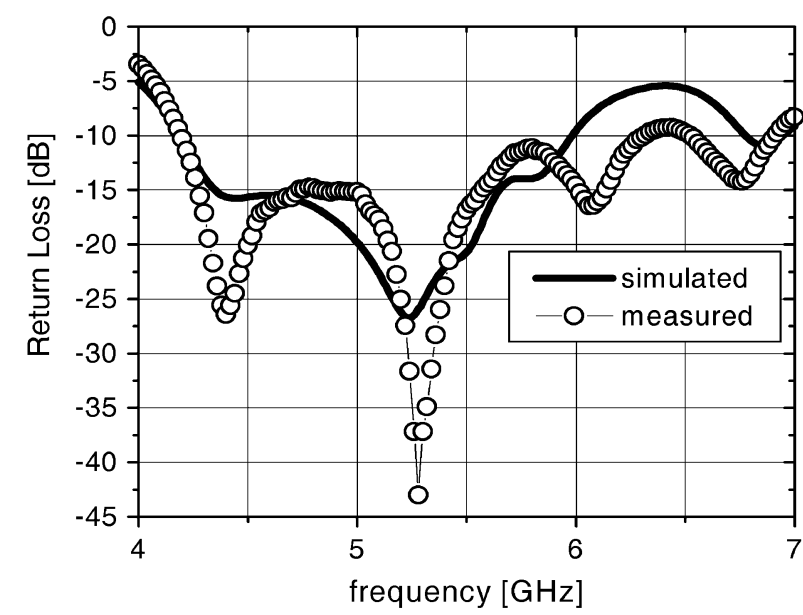

Fig. 2. Measured and simulated return Loss as a function of frequency.

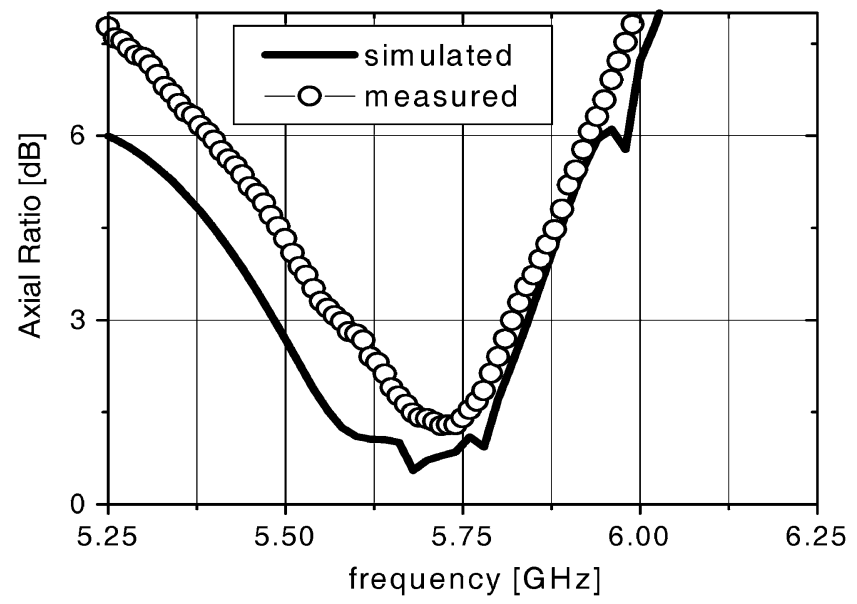

Fig. 3. Measured and simulated axial ratio in broadside direction as a function of frequency.

\section{RESULTS AND DISCUSSION}

According to the specifications given above, an antenna prototype was manufactured. In Fig. 2, the measured and simulated return loss of the cross-slot-coupled DRA is illustrated. The wide impedance bandwidth obtained is due to the slot and DRA resonances. More specifically, the two slots are designed to be resonant at about $4.4 \mathrm{GHz}$ and $5.9 \mathrm{GHz}$ while the $\mathrm{HEM}_{11 \delta}$ mode of the DRA occurs at $5.25 \mathrm{GHz}$.

The axial ratio of the CP operation in the broadside direction ( $z$ axis) was also measured and compared with the simulation (Fig. 3). The measured and simulated CP bandwidth, as determined from the 3-dB axial ratio, was found to be around $4.7 \%$ and $6.3 \%$, respectively. The polarization obtained was left-handed CP since $L_{s 1}>L_{s 2}$.

The simulated data presented in Fig. 4 illustrates the effect of the asymmetric design on the $\mathrm{CP}$ bandwidth of the DRA. In the previously proposed DRA geometry, the ratio $L_{s 1} / L_{s 2}$ is 1.28 . For this ratio, Fig. 4 depicts how the asymmetry of the design ( $x$-axis is the distance $P_{2}-P_{1}$ of the stub lengths) affects the CP bandwidth of the DRA. The effect of the asymmetry on the angle $\theta_{\mathrm{cp}}$ is also shown, where $\theta_{\mathrm{cp}}$ is the angle off broadside direction for which $\mathrm{CP}$ operation is actually obtained (at the center frequency). It is clear that up to a certain point, by increasing the 


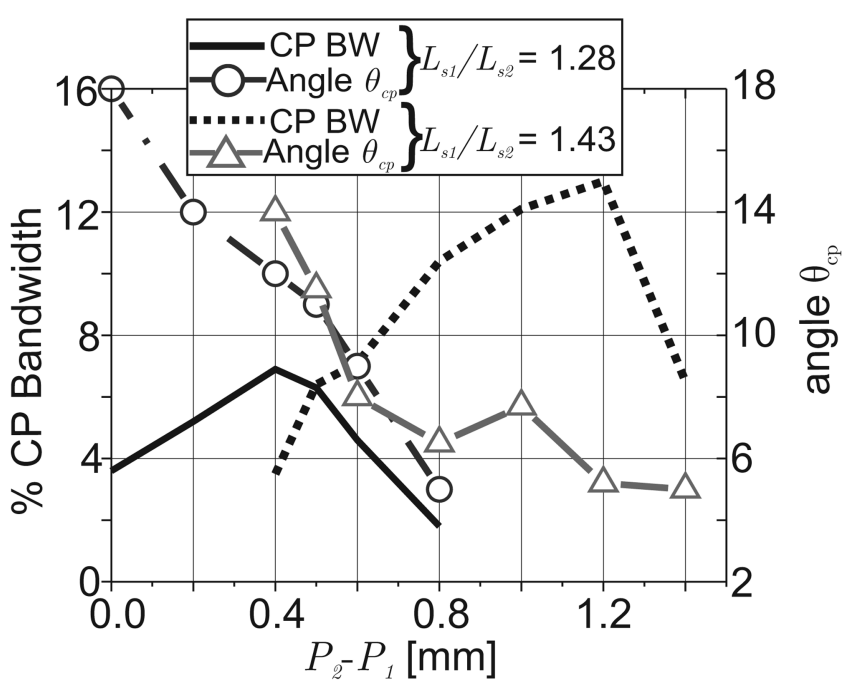

Fig. 4. Simulated CP Bandwidth and angle $\theta_{\mathrm{cp}}$ versus the distance of the centers of the two slots, for two different ratios of slot lengths. $P_{1}=4.2 \mathrm{~mm}$ for $L_{s 1} / L_{s 2}=1.28, P_{1}=3.1 \mathrm{~mm}$ for $L_{s 1} / L_{s 2}=1.43$.

asymmetry, the CP bandwidth becomes broader. On the other side, however, the increasing asymmetry results in a reduction of angle $\theta_{\mathrm{cp}}$ of the $\mathrm{CP}$ operation. As an example, for the case of our manufactured prototype, the stub lengths difference $P_{2}-P_{1}$ is $0.5 \mathrm{~mm}$ and the resulting simulated CP bandwidth is $6.3 \%$ for an angle $\theta_{\mathrm{cp}}$ of about $11^{\circ}$.

If the ratio of the long to the short slot length becomes larger, an even larger broadside CP bandwidth can be obtained, provided that the asymmetry is increased correspondingly. This argument can be further substantiated considering a second set of curves in Fig. 4. The dependence of the design asymmetry on the CP bandwidth and the angle $\theta_{\mathrm{cp}}$ is also shown for the ratio $L_{s 1} / L_{s 2}$ being 1.43 . It is observed that in this case and for $P_{2}=P_{1}$ an axial ratio below $3 \mathrm{~dB}$ cannot be obtained. As the two slot centers are moved apart, $\mathrm{CP}$ bandwidth increases and for a value of $P_{2}-P_{1}$ being about $1.2 \mathrm{~mm}$, the bandwidth becomes maximum. It is important to observe here that the maximum value of CP bandwidth for $L_{s 1} / L_{s 2}=1.43$ is larger than the one for $L_{s 1} / L_{s 2}=1.28$. With respect to the sensitivity on the angle $\theta_{\text {cp }}$, it is obvious that in this case, because of the larger design asymmetry, the angle $\theta_{c p}$ will also be smaller. To test the above, a DRA was designed and manufactured, for which $L_{s 1}=$ $11.7 \mathrm{~mm}, L_{s 2}=8.2 \mathrm{~mm}$ and $P_{1}=3.1 \mathrm{~mm}, P_{2}=4.3 \mathrm{~mm}$. A $6.8 \%$ broadside CP bandwidth was measured, but the angle $\theta_{\mathrm{cp}}$ was reduced to less than $5^{\circ}$.

Finally, the radiation pattern for the first DRA was extracted at $5.75 \mathrm{GHz}$, at which frequency the antenna is circularly polarized. The measured radiation patterns are depicted in Fig. 5 in the orthogonal planes $x-z\left(\phi=90^{\circ}\right)$ and $y-z\left(\phi=90^{\circ}\right)$, respectively. It is apparent that satisfactory left-handed $\mathrm{CP}$ (LHCP) is obtained, with cross polarization down by $-15 \mathrm{~dB}$. At $5.75 \mathrm{GHz}$ the gain in the broadside direction was found to be around $3.5 \mathrm{dBi}$.

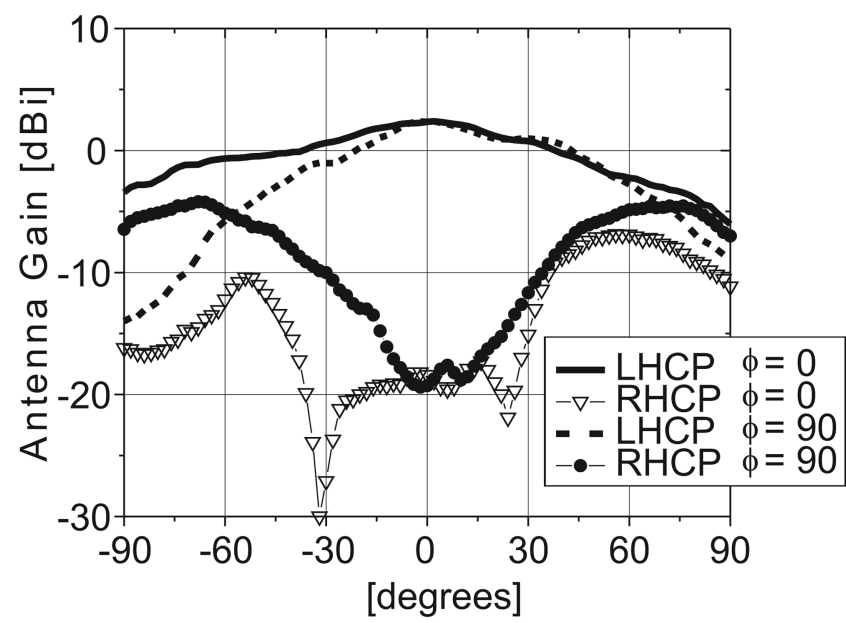

Fig. 5. Measured radiation patterns in the $x-z$ and $y-z$ planes.

\section{CONCLUSION}

A cross-slot-coupled cylindrical dielectric DRA has been described. It was shown that an asymmetric feed structure has a strong influence on the $\mathrm{CP}$ bandwidth without affecting the radiation pattern. By optimizing the slot lengths and the distance of each slot to the open-end of the feeding microstrip line separately, a wide CP bandwidth of $4.7 \%$ is possible. Measurements and simulations are in good agreement.

\section{ACKNOWLEDGMENT}

The authors would like to thank H.-R. Benedickter, for valuable support, and M. Lanz, for fabricating the feeding structure.

\section{REFERENCES}

[1] S. A. Long, M. W. McAllister, and L. C. Shen, "The resonant cylindrical dielectric cavity antenna," IEEE Trans. Antennas Propag., vol. AP-31, no. 3, pp. 406-412, May 1983.

[2] A. Petosa, A. Ittipiboon, Y. M. M. Antar, D. Roscoe, and M. Cuhaci, "Recent advances in dielectric-resonator antenna technology," IEEE Antennas Propag. Mag., vol. 40, no. 3, pp. 35-48, Jun. 1998.

[3] C. S. Hong and C. Y. Huang, "Circularly polarized dielectric resonator antennas," in Proc. Int. Zurich Sem. Broadband Commun., Feb. 2000, pp. 69-71.

[4] A. Laisne, R. Gillard, and G. Piton, "Circularly polarised dielectric resonator antenna with metallic strip," Electron. Lett., vol. 38, no. 3, pp. 106-107, Jan. 2002.

[5] B. Li and K. W. Leung, "Strip-fed rectangular dielectric resonator antennas with/without a parasitic patch," IEEE Trans. Antennas Propag., vol. 53, no. 7, pp. 2200-2207, Jul. 2005.

[6] C. Y. Huang, J. Y. Wu, and K. L. Wong, "Cross-slot-coupled microstrip antenna and dielectric resonator antenna for circular polarization," IEEE Trans. Antennas Propag., vol. 47, no. 4, pp. 605-609, Apr. 1999.

[7] K. K. Pang, H. Y. Lo, K. W. Leung, K. M. Luk, and E. K. N. Yung, "Circularly polarized dielectric resonator antenna subarrays," Microw. Opt. Technol. Lett., vol. 27, no. 6, pp. 377-379, Dec. 2000.

[8] K. M. Luk and K. W. Leung, Dielectric Resonator Antennas. Baldock, U.K.: Research Studies Press, 2003.

[9] A. Buerkle, K. Sarabandi, and H. Mosallaei, "Compact slot and dielectric resonator antenna with dual-resonance, broadband characteristics," IEEE Trans. Antennas Propag., vol. 53, no. 3, pp. 1020-1027, Mar. 2005. 